
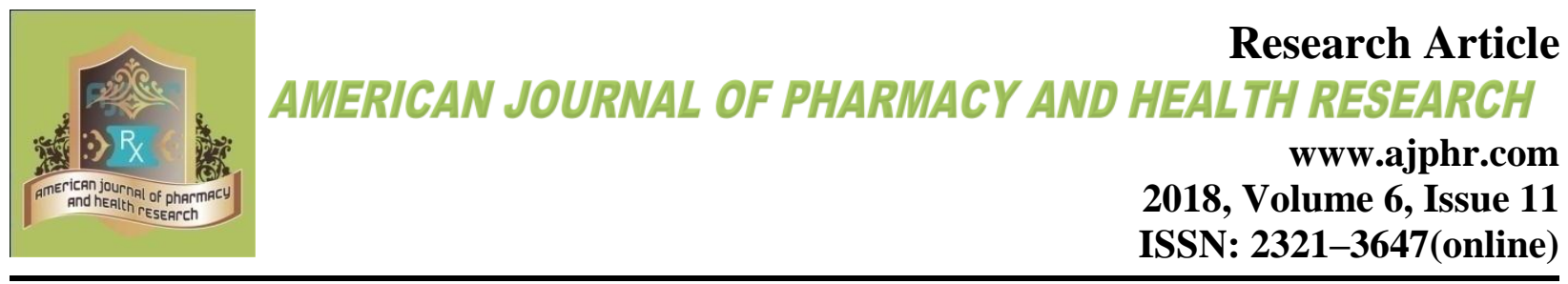

\title{
Case Study on Management of T.B Meningitis
}

\author{
Brahma Reddy D.R*, Satyanarayana V, Bhavani M, Suresh Kumar M, Tejaswi R, \\ Sharon S. \\ Nalanda Institute of Pharmaceutical Sciences, Kantepudi (V), Sattenapalli (M), Guntur (Dt), \\ Andhra Pradesh, India.
}

\begin{abstract}
In this paper we present a case of a 9 year old male patient with a rare form of extra pulmonary tuberculosis-tuberculous meningitis. Meningitis is an inflammation of the meningeal layers of the brain and spinal cord. Different types of meningitis have been determined, each with its own causes, risk factors and side effects. Here are the five most common types of meningitis, they are Viral Meningitis, Bacterial Meningitis, Parasitic Meningitis, Fungal Meningitis, Chronic Meningitis. TBM is a rare form of extra pulmonary tuberculosis.
\end{abstract}

Keywords: extra pulmonary tuberculosis, tuberculous meningitis.

*Corresponding Author Email: desireddybrahma@ rediffmail.com Received 10 September 2018, Accepted 20 October 2018

\section{INTRODUCTION}

Please cite this article as: Reddy B et al., Case Study on Management of T.B Meningitis .American Journal of Pharmacy \& Health Research 2018. 
Meningitis is an inflammation of the meningeal layers of the brain and spinal cord. The infection involves the arachnoid the pia mater, and the intruding cerebrospinal fluid (CSF). The inflammatory process expands throughout the subarachnoid space of the brain and spinal cord and include the ventricles. Meningitis can cause necrosis, reduced blood and CSF flow, and impaired central nervous system (CNS) function. The early symptoms are headache, fever, and chills; however, a combination of 2 to 4 symptoms (headache, fever, stiff neck and varied mental status) is found in $95 \%$ of patients. Death occurs from shock of the emergence of symptoms $[1,2]$.

Different types of meningitis have been resolved, each with its own causes, risk factors and side effects. Here are the five most common types of meningitis $[3,4]$.

\section{Viral Meningitis}

It said to be the most common type of meningitis, viral meningitis occurs because certain viruses enter into your body. However it is similar to bacterial meningitis in terms of the symptoms they exhibit, viral meningitis is different because it's an restrained illness. This means that bacteria won't grow in the cerebrospinal fluid, which is a clear and colourless liquid that surrounds your brain and spinal cord $[5,6]$.

\section{Bacterial Meningitis}

Bacterial meningitis is caused by certain bacteria strains that enter your blood stream and go further into your brain and spinal cord. Sometimes, this disease could also arise because of an ear or sinus infection, a skull fracture or as side effect of surgery (although this is rare) [7].The identified bacteria strains that cause this disease are [8]:

- Streptococcus pneumoniae: This bacteria strain is distinctively located in the respiratory tract, sinuses and nasal cavity, and may cause pneumococcal meningitis.

- Neiserria meningitides: Mainly spread through the saliva and other respiratory fluids, Neiserria meningitides could trigger meningitis, septicaemia or a combination of both illnesses. This bacteria own other strains such A, B, C, W, X and Y, [9] with B being the most common type, causing meningitis B [10].

- Haemophilus influenza: A very threatening bacteria strain, this infection not only results in the start of meningitis, but can also cause blood infection/s, wind pipe inflammation, cellulitis and infectious arthritis.

- Listeria monocytogenes: This is a type of bacteria that can taint various food items and may lead to an infection called listeriosis [11]. 
- Mycobacterium tuberculosis[12]: This strain ultimately causes tuberculous (TB) meningitis in a patient. The infection begins in the lungs or anywhere in the body.

\section{Parasitic Meningitis}

This is a type of meningitis where in a patient is infected with Naegleria fowleri. This is a Parasite that's said to cause primary amebic meningo encephalitis (PAM)[13].

Nevertheless, other parasites can also cause an illness called eosinophilic meningitis, eosinophilic meningo encephalitis or EM, where in high levels of eosinophils are documented. Eosinophils are a type of white blood cell in the cerebrospinal fluid around your brain and spinal cord.

\section{Fungal Meningitis}

If you have fungal meningitis, this means that a fungus has invaded the blood stream and has moved through the blood and into your spinal cord [14]. This type of meningitis could appear after you take medications that enfeeble your immune system [15]. In fact, while practically everyone is at risk of having fungal meningitis, your risk becomes far up if you have a weak immune system [16]. While this disease is not passed from person to person, there are many ways that the fungus responsible for this illness can be transmitted, namely [17]:

- Cryptococcus: Considered to be the most common type of fungus that causes fungal meningitis, you can acquire this when you inhales oil that has findings of bird droppings.

- Hitoplasma: This type of fungus is found in environments with huge amounts of bird or bat droppings, such as in the Midwest, near the Ohio and Mississippi rivers.

- Blastomyces: This is located in soil that is rich in decaying organic matters, specifically in the northern Mid west area here in the U.S. Non-Infectious Meningitis: Certain diseases (cancer or lupus), procedures (brain surgery), injuries (head injuries) or medications can cause non-infectious meningitis. Just like parasitic and fungal meningitis, this illness cannot be carried from one person to another [18].

\section{Chronic Meningitis}

If you experience a condition with meningitis-like symptoms that evolve over weeks and months, chances are you have chronic meningitis. Symptoms like fever, headaches, confusion, facial numbness and incidences of paralysis are common in this disease, however they don't manifest as quickly and aren't that severe [19]. People who are immune compromised because of diseases like cancer and AIDS, cancer treatment medications and long-term drug use (particularly prednisone) are more likely to have chronic meningitis [20]. 


\section{CASE REPORT:}

A 9 yr old male child was admitted to the paediatric intensive care unit of Ala Hospital with chief complaints of fever, vomiting, headache. He was vaccinated with BCG as a child. Patient did not have any medical problems. There was no family history of T.B. He underwent CT-scan of head, the report was normal and he underwent lumber puncture (LP) simultaneously. The Cerebrospinal fluid analysis shows that the total cell count was raised and sediment cytology reveals that many lymphocytes along with few neutrophils and also observed that occusional RBC may also found. CSF for ADA raised to 11.2U/L (reference range : $>10$ is positive). Pandys test was given positive and there is decrease in glucose level to $44 \mathrm{mg} / 100 \mathrm{ml}$ (reference range: 60 to $\mathrm{mg} / 100 \mathrm{ml}$ ). Hence, final diagnosis was made as TB meningitis.

Patient was treated with Inj Monocef 750mg Iv BID, Inj Vancomycin 500mg IV BD for 7 days, Inj Mannitol-50ml Iv 6th hourly for 7 days, Inj Rantac-1CC Iv BD for 7 days, Tab Dolo-650mg TID for 7 days along with fluid and other supportive measures for 7 days and was discharged after 7 days of hospital stay. The patient attenders were counselled and his discharge medications were Inj Accuzon-250mg BD, Inj Vancomycin-500mg-BD, Inj Rantac-1CC BD for a week.

\section{RESULTS AND DISCUSSION}

TBM is a rare form of extra pulmonary tuberculosis [21]. The disease infests slowly, and is difficult to diagnose, because it does not manifest itself initially with characteristic symptoms. In the discussed study the disease progressed in 3 different forms of TBM phases [21, 23]. The condition of the patient degenerate significantly with the emergence of subsequent symptoms proving the improvement of the disease, and the prognosis for total recovery, without significant neurological deficits depended on the time of anti tuberculotic treatment implementation. In differential diagnosis different etiological factors were taken into inclusion, which could have caused tuberculous meningitis and encephalitis (viruses, bacteria)

CSF test plays a major role in suspected tuberculosis etiology. CSF lymphocytosis, increased concentration of proteins, and decreased concentration of CSF glucose suggest diagnosis. In CSF examination at the beginning of the patients hospitalisation, in addition to typical variation in protein and glucose levels [24]. Glucose concentration in CSF (44mg/100ml) fully met the criterion level of less than $70 \%$ of the concentration in the blood serum. The growth of Mycobacterium tuberculosis in culture of the CSF sample is still the best standard way in diagnosis of tuberculosis. Regrettably, traditional microbiological tests have low sensitivity and long waiting 
period for positive results, while new and faster molecular tests (bacterial DNA amplification by PCR) remain costly and isolated in routine hospital diagnosis. Although these tests have positive predictive value and high specificity, their sensitivity in diagnosis of extra- pulmonary tuberculosis is hardly gratified. This is most likely due to low concentration of mycobacterium in CSF, inadequate volume of test material (CSF), and the presence of inhibitory substances in the process of bacterial DNA amplification. Due to this, negative test results do not exempt TBM. Taking into account the above mentioned factors, molecular tests should be conducted along with the traditional microbiological studies [25, 26]. Bacteriological infection confirmation and thus defined time necessary to obtain tubercular mycobacteria growth, (depending on medium, sometimes up to 2 months) should not delay appropriate therapy if there is a clear clinical rationale suggestive of TBM. Imagining tests (CT, MR) of CNS are helpful in identifying the seriousness of the disease, and qualification of the patients for life saving medical procedures (severe hydrocephalus). In the course of TBM CT scan of the head usually reveals in at least half abnormalities on the base of the brain and cerebral infarctions. Less frequently tuberculoma, or oedema of the brain are observed. MR of the head is more sensitive in imaging of CNS inflammation lesions (meningeal enhancement) in the TBM compared to CT scan [27, 28]. In approximately $50 \%$ of patients with TB there are also tuberculosis lesions in the lungs, so x-ray of the chest as well as microbiological sputum test, remain an essential part in diagnostic process. On a basic of the chest x-ray and patients history, it was decided to commence specific therapy. The choice of right combination of antituberculotic drugs as well time of TBM treatment, should cover intensive 4x drug treatment (Isoniazid INH, Rifampicin RMP, Pyrzinamid PZA, and Streptomycin $\mathrm{SM}$ ), which continuation will be two drug maintenance therapy (INH+RMP) for 12 months. Administration of glucocorticoids (Dextamethasone) favourably influences revisable changes in CSF as well as greatly increases survival of patients. They should be administered sequentially over a period of 6-8 weeks in decreasing doses. As the patient is very young and it is a very long waiting period for the diagnosis of TBM, the standard treatment was skipped and the patient was treated with Inj Monocef 750mg Iv BID, Inj Vancomycin 500mg IV BD, Inj Mannitol-50ml Iv 6th hourly, for 7 days Inj Rantac-1CC Iv BD Tab Dolo-650mg TID along with fluid and other supportive measures for 7 days and was discharged after 7 days of hospital stay. The patient attenders were counselled and his discharge medications were Inj Accuzon-250mg BD, Inj Vancomycin-500mg-BD, Inj Rantac-1CC BD for a week. 
T.B meningitis is a large public health concern. Continuous diagnosis improvement is necessary to minimize disease mortality and life- threatening complications. Continued updates to describe prevalence and expanded reporting of outbreaks will help to evaluate vaccination outcomes and impact. Rational utilization of drugs plays a major role in the management of T.B meningitis.

\section{REFERENCE}

1. Matthew, P.F. The nervous system, Robbins basic pathology 2007; 873-881.

2. Morton, N.S. and A. Mitchell Meningitis: bacterial, viral, and other in Lee Goldman (ed), Goldman's Cecil medicine, Saunders Elsevier, Philadelphia, PA. 2012; 2355-2370.

3. Theobald and Bass, "Understanding the 5 Types of Meningitis," Everyday Health, July18, 2014.

4. Lights, Boskey, and Krucik, "Meningitis”, Healthline, January 12, 2016.

5. Theobald and Bass, "Understanding the 5 Types of Meningitis," Everyday Health, July18, 2014.

6. “Cerebrospinal Fluid (CSF)" National Multiple Sclerosis Society.

7. Mayo Clinic Staff, "Meningitis Symptoms and Causes”, Mayo Clinic, January 12, 2016.

8. Lights, Boskey, and Krucik, “Meningitis”, Healthline, January 12, 2016.

9. “Meningococcal disease, ”Meningitis Research Foundation.

10. Sadarangani, "What Is Meningitis B - and Why Don't Older Children Getthe Vaccine?", The Conversation, February 24, 2016.

11. "Listeria (Listeriosis) Definitions \& Symptoms,"Centers for Disease Control and Prevention, November 20, 2014.

12. “Tuberculous (TB) Meningitis The Facts,”Meningitis Trust, July 2013.

13. Theobald and Bass, "Understanding the 5 Types of Meningitis," Everyday Health, July18, 2014.

14. "Fungal Meningitis", Centers for Disease Control and Prevention, April 15, 2016.

15. "Fungal Meningitis", Centers for Disease Control and Prevention, April 15, 2016.

16. Theobald and Bass, "Understanding the 5 Types of Meningitis", Every day Health, July $18,2014$.

17. “Fungal Meningitis,”Center's for Disease Control and Prevention, April 15, 2016.

18. Theobald and Bass, "Understanding the 5 Types of Meningitis", Everyday Health, July 18, 2014. 
19. "Meningitis",Clevel and Clinic, November 28, 2014.

20. "Meningitis",Clevel and Clinic, November 28, 2014.

21. DOTS Expansion Plan to Stop TB in the WHO European Region 2002-2006. World Health Organization. Regional Office for Europe 2002.

22. Coyle PK. Glucocorticoids in central nervous system bacterial infections. Arch Neurl 1999; 56:796-801.

23. Prusinski A. Neurologia praktyczna, Wyd.2 Warszawa: Wydaw Lek PZWL; 2001: 188189.

24. Wojewódzka Stacja Sanitarno-Epidemiologiczna w Poznaniu, http://wsse-poznan.pl/wpcontent/up- loads/2011/05/PlikLeczenie.pdf

25. Pai M, Ramsay A, O. Brien R Evidence-Based Tuber- culosis Diagnosis. PLoS Med 5(7): e156. Doi: 10.1371/ journal. PMED.

26. Pai M, Flores LL, Pai N. Diagnostic accuracy of nucleic acid amplification tests for tuberculous meningitis: a systematic review and meta-analysis. Lancet Infect Dis 2003; 3(10):633-43.

27. Chotmongkol V, Panthavasit J, Tiamkao S. Tuberculous meningitis in adults: a four-year review during 1997-2000. Southeast Asian J Trop Med Public Health 2003; 34(4):869-871.

28. Christensen ASH, Andersen AB, Thomsen V. Tuberculous meningitis in Denmark: a review of 50 cases. BMC Infect Dis 2011; 11:47.

\section{AJPHR is}

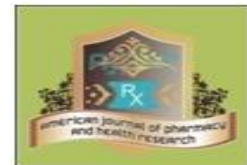

Peer-reviewed

monthly

Rapid publication

Submit your next manuscript at editor@ajphr.com / editor.ajphr@gmail.com 